\title{
Morfometría comparada de los estatolitos del calamar Loligo gahi d'Orbigny, 1835 (Cephalopoda: Loliginidae) del norte de Perú e islas Falkland
}

\author{
Marco A. Vega ${ }^{1,2}$, Francisco J. Rocha ${ }^{2}$ y Cecilia Osorio ${ }^{1}$ \\ ${ }^{1}$ Laboratorio de Hidrobiología, Facultad de Ciencias, Universidad de Chile \\ Casilla 653, Santiago, Chile. E-mail: mvega @iim.csic.es, E-mail: cosorio@uchile.cl \\ ${ }^{2}$ Instituto de Investigaciones Marinas (CSIC) \\ C/ Eduardo Cabello 6, 36208 Vigo, España. E-mail: frocha@iim.csic.es
}

Recibido: 19 noviembre 1999; versión corregida: 14 julio 2000; aceptado: 2 noviembre 2000

\begin{abstract}
RESUMEN. Se analizó un total de 241 estatolitos: 141 pertenecientes a Loligo gahi del norte del Perú (70 estatolitos izquierdos y 71 derechos) correspondientes a 34 machos y 47 hembras, y 100 de las islas Falkland (50 estatolitos izquierdos y 50 derechos) correspondientes a 35 machos y 23 hembras. En cada estatolito se midió longitud total (LT), longitud del domo (LD) y longitud del rostro (LR), determinándose en cada ejemplar la longitud dorsal del manto (LM) y sexo. Las medidas se transformaron como cocientes de LM, comparándose mediante análisis discriminante. No se observaron diferencias significativas entre los estatolitos izquierdo y derecho en ambos sexos y en ambas áreas, por lo que se compararon sólo los del lado derecho. Se hallaron diferencias significativas entre machos y hembras del norte de Perú así como entre ambos sexos de las islas Falkland. También se hallaron diferencias significativas para individuos del mismo sexo entre ambas zonas. Se discute la significación de las diferencias encontradas entre los estatolitos de L. gahi del Perú e islas Falkland.
\end{abstract}

Palabras claves: Loligo gahi, estatolitos, morfometría, análisis discriminante, Perú, islas Falkland.

\section{Compared morphometry of squid statoliths Loligo gahi d'Orbigny, 1835 (Cephalopoda: Loliginidae) of northern Perú and Falkland islands}

\begin{abstract}
A total of 241 statoliths were analyzed: 141 belonging to Loligo gahi from the Northern Perú (70 left statoliths and 71 right statoliths) corresponding to 34 males and 47 females, and 100 from the Falkland Islands (50 left statoliths and 50 right statoliths) corresponding to 35 males and 23 females. Total length (TL), dome length (DL) and face length (FL) were measured, determining in each specimen the mantle dorsal length (ML) and sex. The measures became quotients of ML, and were compared using discriminant analysis. Significant differences between the left statolith and the rigth statolith were not observed in either sex, reason why those of the right hand side were compared. Significant differences between males and females from the North of Perú were found as well as between both sexes from those of the Falkland Islands. There were also significant differences for individuals of the same sex between both zones. The meaning of the differences found between L. gahi statoliths from Perú and Falkland Islands are being discussed.
\end{abstract}

Key words: Loligo gahi, statoliths, morphometry, discriminating analysis, Perú, Falkland Islands.

\section{INTRODUCCIÓN}

El calamar patagónico, Loligo gahi d'Orbigny, 1835, se captura en las costas de los océanos Pacífico y Atlántico desde el Perú hasta la Argentina, soportando una importante pesquería internacional en la plataforma de la Patagonia (Roper et al., 1984; Anónimo, 1989; FAO, 1997). En el área alrededor de las islas Falkland esta especie constituye uno de los mayores recursos económicos, alcanzando un máximo de 300 millones de dólares en 1987 (Patterson, 1988; Anónimo, 1989). En el océano Pacífico suroriental, el calamar patagónico no representa un recurso importante, capturándose incidentalmente en las pesquerías artesanales y de arrastre de Perú y Chile. Las capturas de este molusco, en ambos paí- 
ses, no superan las 8000 y 500 toneladas anuales, respectivamente (FAO, 1997).

Aunque L. gahi es una especie bastante estudiada en el área de las Falkland (Hatfield y Des Clers, 1998), poco se sabe de ella y de las poblaciones que la componen en el área del Pacífico sudeste. Incluso algunos autores, como Nesis (1987), indican que realmente existen dos especies: una en el océano Pacífico (L. gahi) y otra en el Atlántico (Loligo patagonica Smith, 1881). Aclarar la identidad de esta especie e identificar sus unidades de población es de suma importancia, tanto para el estudio de su biología y ecología, como para el futuro manejo del recurso en las pesquerías del área.

El estudio de los estatolitos, estructuras calcáreas pares que se alojan en los estatocistos en la región posteroventral del cartílago craneal, representa una alternativa válida para la identificación de especies y delimitación de poblaciones en los cefalópodos (Clarke, 1978; Clarke et al., 1980; Clarke y Maddock, 1988; Leta, 1982; Lipinski et al., 1993; Pineda et al., 1998). La comparación de la morfometría de los ejemplares o de alguna de sus estructuras más estables, es uno de los métodos más tradicionales y reconocidos en los estudios sistemáticos y para la discriminación entre especies o la discriminación entre stocks (Ihssen et al., 1981; George-Nascimento y Arancibia, 1992; Borges, 1995).

En el presente trabajo se realiza una primera aproximación al estudio y comparación de las poblaciones de Loligo gahi del norte de Perú e islas Falkland mediante el análisis morfométrico comparado de sus estatolitos.

A

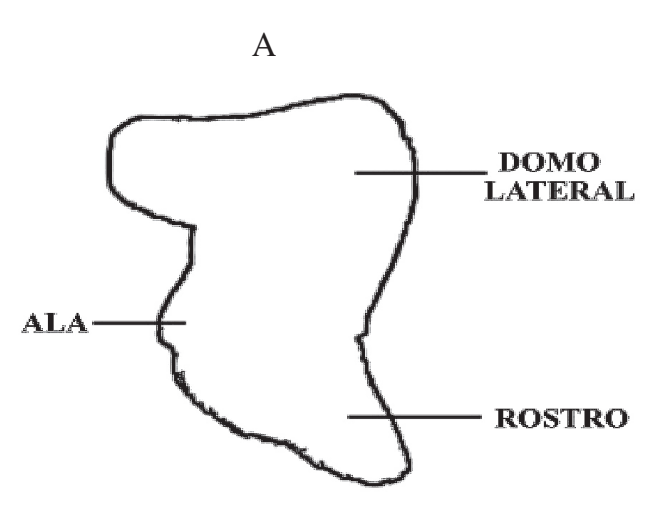

\section{MATERIALES Y MÉTODOS}

Se analizó un total de 241 estatolitos: 141 pertenecientes a ejemplares del norte del Perú (70 estatolitos izquierdos y 71 derechos), correspondientes a 34 machos y 47 hembras, y 100 de islas Falkland (50 estatolitos izquierdos y 50 derechos), pertenecientes a 35 machos y 23 hembras. Las muestras de las islas Falkland fueron obtenidas en abril de 1997 de las capturas desembarcadas en Punta Arenas por Pesca Chile S.A., mientras que los ejemplares del norte del Perú se obtuvieron en octubre de 1997 a través de BCA Comercial Ltda. (Santiago). Los ejemplares fueron congelados a bordo de los pesqueros después de su captura y conservados a $20^{\circ} \mathrm{C}$. Las muestras fueron transportadas al laboratorio en neveras portátiles a $-18^{\circ} \mathrm{C}$. Una vez allí se conservaron a $-20^{\circ} \mathrm{C}$ hasta su análisis. Una vez descongelados a temperatura ambiente, a cada ejemplar se le determinó la longitud dorsal del manto (LM), mediante un pie de metro de $0,01 \mathrm{~mm}$, y el sexo. Los estatolitos se extrajeron utilizando las técnicas descritas por Clarke (1978) y Arkhipkin (1990). Posteriormente, fueron conservados en alcohol etílico al $95 \%$ hasta ser medidos. Todos los estatolitos fueron medidos por la misma persona bajo un microscopio binocular Zeiss provisto de ocular micrométrico. Las medidas y términos utilizados se basaron en Clarke (1978) y Guerra y Sánchez (1985) (Fig. 1). Las medidas realizadas se expresan en milímetros (mm) y fueron las siguientes: longitud total (LT), longitud del domo (LD) y longitud del rostro (LR). Dos medidas, la longitud del domo dorsal y la longitud del ala, fueron descartadas, debido a la

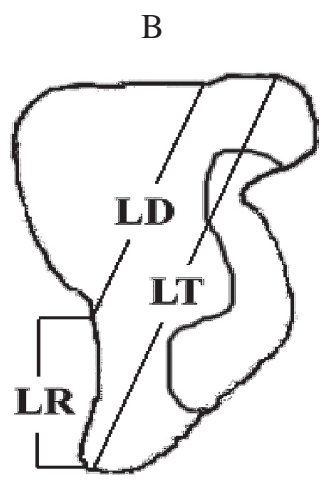

Figura 1. Estatolito derecho de Loligo gahi. A. Vista posterior, partes principales. B. Vista anterior, mediciones realizadas: longitud total (LT), longitud del domo (LD), longitud del rostro (LR).

Figure 1. Loligo gahi right statolith. A. Posterior view showing the main structures. B. Anterior view showing the main morfometric measurements: total length (LT), dome length (LD), rostrum length (LR). 
imposibilidad de definir con precisión sus límites.

Para la detección de cualquier dato incoherente y probar el supuesto de normalidad de los datos se realizó un análisis de los residuos de la regresión de cada carácter morfométrico frente a la longitud dorsal del manto (Canavos, 1996; Gujarati, 1997). Para cada variable morfométrica se estimó su valor promedio, rango de variación y desviación estándar. Todas las variables medidas se expresaron como fracción de la longitud dorsal del manto (LM) para evitar superposición de tallas entre grupos (Pineda et al., 1996), utilizándose estos cocientes para las comparaciones realizadas entre ambas áreas.

Con objeto de comprobar si existían o no diferencias significativas entre los estatolitos derecho e izquierdo de cada ejemplar, éstos se compararon considerando cada sexo y localidad por separado mediante un test de significancia para la distribución $t$ de Student (Manly, 1994). Posteriormente, se compararon los estatolitos de cada sexo para una misma localidad y entre localidades para cada sexo, utilizándose un análisis discriminante (Cuadra, 1989). Todos los análisis se realizaron con el programa Statistica, 5.0.

\section{RESULTADOS}

Las Tablas 1 y 2 presentan las medias, desviaciones estándares y rangos de variación de las medidas tomadas y como fracción porcentual de la longitud dorsal del manto de los estatolitos izquierdos y derechos de machos y hembras del norte de Perú e islas Falkland con su rango y desviación estándar. Dado que no se observaron diferencias significativas $(p>0,001)$ entre los estatolitos izquierdo y derecho, tanto en machos como en hembras del norte del Perú e islas Falkland, las comparaciones entre los ejemplares de ambas áreas se realizaron considerando solamente los estatolitos del lado derecho.

Las Tablas 3 y 4 muestran los resultados del análisis discriminante para machos y hembras del norte de Perú e islas Falkland. La Tabla 3a muestra que hay diferencias significativas entre machos y hembras de Perú y entre machos y hembras de las islas Falkland. Asimismo, se hallaron diferencias significativas entre machos del norte del Perú e islas Falkland y entre las hembras de ambas localidades (Tabla 3b). En la Tabla 4 se indica el peso de las variables utilizadas para cada una de las comparaciones.

Tabla 1. Estadística básica de las medidas de los estatolitos izquierdo y derecho, y cociente $(\%)$ de longitud dorsal de machos y hembras de Loligo gahi del norte del Perú.

Table 1. Statistics basic of the measures of the left and right hand side statoliths and quotient (\%) of dorsal length of the Loligo gahi male and female mantle from the north of Perú.

\begin{tabular}{|c|c|c|c|c|c|c|c|c|}
\hline Sexo & Variable & $\begin{array}{c}\mathrm{N}^{\circ} \\
\text { Estatolitos }\end{array}$ & $\begin{array}{c}\text { Media } \\
(\mathrm{mm})\end{array}$ & $\begin{array}{c}\text { Rango } \\
(\mathbf{m m})\end{array}$ & D.S. & $\begin{array}{l}\text { Media } \\
\text { \% LM }\end{array}$ & Rango & D.S. \\
\hline \multicolumn{9}{|l|}{ Estatolito Izquierdo } \\
\hline \multirow{4}{*}{ Machos (N=34) } & LT & 30 & 1,59 & $1,45-1,72$ & 0,07 & 1,06 & $0,83-1,34$ & 0,14 \\
\hline & $\mathrm{LD}$ & 30 & 0,90 & $0,84-0,97$ & 0,04 & 0,60 & $0,45-0,78$ & 0,08 \\
\hline & LR & 30 & 0,53 & $0,44-0,66$ & 0,05 & 0,35 & $0,28-0,47$ & 0,05 \\
\hline & LM & 30 & 153 & $113-201$ & 22 & & & \\
\hline \multirow[t]{4}{*}{ Hembras $(\mathrm{N}=47)$} & LT & 40 & 1,58 & $1,50-1,67$ & 0,04 & 1,06 & $0,92-1,19$ & 0,07 \\
\hline & LD & 40 & 0,89 & $0,86-0,99$ & 0,03 & 0,60 & $0,51-0,71$ & 0,05 \\
\hline & LR & 40 & 0,54 & $0,46-0,64$ & 0,04 & 0,36 & $0,29-0,46$ & 0,04 \\
\hline & LM & 40 & 150 & $130-175$ & 12 & & & \\
\hline \multicolumn{9}{|l|}{ Estatolito Derecho } \\
\hline \multirow[t]{4}{*}{$\operatorname{Machos}(\mathrm{N}=34)$} & LT & 29 & 1,60 & $1,47-1,78$ & 0,07 & 1,06 & $0,82-1,33$ & 0,14 \\
\hline & LD & 29 & 0,90 & $0,84-0,97$ & 0,03 & 0,59 & $0,45-0,78$ & 0,08 \\
\hline & LR & 29 & 0,52 & $0,44-0,66$ & 0,05 & 0,34 & $0,27-0,46$ & 0,06 \\
\hline & LM & 29 & 156 & $113-201$ & 23 & & & \\
\hline \multirow[t]{4}{*}{ Hembras $(\mathrm{N}=47)$} & LT & 42 & 1,59 & $1,52-1,72$ & 0,05 & 1,07 & $0,91-1,23$ & 0,08 \\
\hline & LD & 42 & 0,89 & $0,84-0,95$ & 0,02 & 0,60 & $0,51-0,69$ & 0,04 \\
\hline & LR & 42 & 0,54 & $0,44-0,64$ & 0,05 & 0,36 & $0,28-0,44$ & 0,04 \\
\hline & LM & 42 & 150 & $130-175$ & 12 & & & \\
\hline
\end{tabular}

LT: Longitud total; LD: Longitud del domo; LR: Longitud del rostro; LM: Longitud dorsal del manto; N: Número de individuos. 
Tabla 2. Estadística básica de las medidas de los estatolitos izquierdo y derecho y cociente (\%) de longitud dorsal de machos y hembras de Loligo gahi de islas Falkland.

Table 2. Statistics basic of the measures of the left and right hand side statoliths and quotient (\%) of dorsal length of the Loligo gahi male and female mantle from the Falkland Islands.

\begin{tabular}{|c|c|c|c|c|c|c|c|c|}
\hline Sexo & Variable & $\begin{array}{c}\mathbf{N}^{\circ} \\
\text { Estatolitos }\end{array}$ & $\begin{array}{c}\text { Media } \\
(\mathrm{mm})\end{array}$ & $\begin{array}{c}\text { Rango } \\
(\mathbf{m m})\end{array}$ & D.S. & $\begin{array}{l}\text { Media } \\
\% \text { LM }\end{array}$ & Rango & D.S. \\
\hline \multicolumn{9}{|l|}{ Estatolito Izquierdo } \\
\hline \multirow[t]{4}{*}{ Machos $(\mathrm{N}=35)$} & LT & 33 & 1,73 & $1,58-1,90$ & 0,08 & 1,11 & $0,96-1,22$ & 0,07 \\
\hline & LD & 33 & 0,93 & $0,86-1,01$ & 0,04 & 0,60 & $0,51-0,66$ & 0,04 \\
\hline & LR & 33 & 0,64 & $0,55-0,75$ & 0,04 & 0,41 & $0,33-0,46$ & 0,03 \\
\hline & LM & 33 & 156 & $134-187$ & 11 & & & \\
\hline \multirow[t]{4}{*}{ Hembras (N=23) } & LT & 17 & 1,75 & $1,67-1,87$ & 0,06 & 1,17 & $1,08-1,27$ & 0,06 \\
\hline & LD & 17 & 0,93 & $0,86-0,99$ & 0,05 & 0,62 & $0,56-0,68$ & 0,04 \\
\hline & LR & 17 & 0,64 & $0,57-0,70$ & 0,04 & 0,43 & $0,38-0,48$ & 0,03 \\
\hline & LM & 17 & 150 & $139-167$ & 7 & & & \\
\hline \multicolumn{9}{|l|}{ Estatolito Derecho } \\
\hline \multirow{4}{*}{ Machos (N=35) } & LT & 34 & 1,74 & $1,56-1,90$ & 0,08 & 1,11 & $0,96-1,21$ & 0,07 \\
\hline & LD & 34 & 0,93 & $0,88-1,01$ & 0,04 & 0,60 & $0,49-0,66$ & 0,04 \\
\hline & LR & 34 & 0,64 & $0,55-0,73$ & 0,04 & 0,41 & $0,35-0,46$ & 0,03 \\
\hline & LM & 34 & 156 & $134-187$ & 11 & & & \\
\hline \multirow[t]{4}{*}{ Hembras (N=23) } & LT & 16 & 1,76 & $1,65-1,89$ & 0,07 & 1,18 & $1,07-1,32$ & 0,07 \\
\hline & LD & 16 & 0,94 & $0,88-1,03$ & 0,05 & 0,63 & $0,58-0,74$ & 0,04 \\
\hline & LR & 16 & 0,65 & $0,57-0,73$ & 0,05 & 0,43 & $0,36-0,50$ & 0,04 \\
\hline & LM & 16 & 150 & $139-164$ & 7 & & & \\
\hline
\end{tabular}

LT: Longitud total; LD: Longitud del domo; LR: Longitud del rostro; LM: Longitud dorsal del manto; N: Número de individuos.

Tabla 3. Resultados del análisis discriminante de las medidas del estatolito de Loligo gahi, por sexos y por áreas. Table 3. Results of the discriminant analysis of the Loligo gahi statolith measures, by sex and by area.

A. Entre sexos para el norte del Perú y para las islas Falkland.

\begin{tabular}{|ccccccc|}
\hline Población & Grupos & Variables & Lambda Wilks & Valor F & Gl & $\mathrm{p}$ \\
\hline Norte de Perú & Machos vs Hembras & 3 & 0,95 & $1,47^{*}$ & 3,66 & $\mathrm{p}<0,23$ \\
Islas Falkland & Machos vs Hembras & 3 & 0,81 & $3,54^{* *}$ & 3,46 & $\mathrm{P}<0,02$ \\
\hline
\end{tabular}

B. Entre áreas para machos y para hembras

\begin{tabular}{|cccccccc|}
\hline Sexo & Grupos & Variables & Lambda Wilks & Valor F & Gl & $\mathrm{p}$ \\
\hline Machos & Perú vs Falkland & 3 & 0,50 & $20,01^{* * *}$ & 3,59 & $\mathrm{p}<0,00001$ \\
Hembras & Perú vs Falkland & 3 & 0,54 & $15,22^{* * *}$ & $3, \quad 53$ & $\mathrm{p}<0,00001$ \\
\hline
\end{tabular}

$*$ Diferencias significativas $(\mathrm{p}<0,2) ; *$ Diferencias significativas $(\mathrm{p}<0,01)$; *** Diferencias significativas $(\mathrm{p}<0,0001)$. 
Tabla 4. Coeficientes estandarizados de las variables canónicas del análisis discriminante entre sexos para una misma área y entre áreas para un mismo sexo.

Table 4. Standardized coefficients of the canonical variables of the discriminant analysis between sexes for the same area and between areas for the same sex.

\begin{tabular}{|ccc|ccc|}
\hline \multicolumn{2}{|c|}{ Entre sexos para una misma área } & \multicolumn{3}{c|}{ Entre áreas para un mismo sexo } \\
\hline Variable & Perú & Falkland & Variable & Machos & Hembras \\
LT & 1,527 & 0,567 & LT & $-0,255$ & 1,324 \\
LD & $-0,406$ & 0,427 & LD & $-0,677$ & $-1,068$ \\
LR & $-1,716$ & 0,064 & LR & 1,411 & 0,505 \\
Valor propio & 0,056 & 0,231 & Valor propio & 0,984 & 0,862 \\
R canónico & 0,229 & 0,433 & R canónico & 0,704 & 0,680 \\
\end{tabular}

El porcentaje correcto de ejemplares clasificados entre machos y hembras de Perú y entre ambos sexos de islas Falkland, fue relativamente bajo $(63,8 \%$ y 76,0 respectivamente), siendo más alto cuando se compararon entre machos de Perú y Falkland (82,3\%) $\mathrm{y}$ entre hembras de ambas zonas $(85,9 \%)$.

\section{DISCUSIÓN}

Dadas las limitaciones del muestreo no se pudo obtener un tamaño mayor de muestra ni recolectar simultáneamente ejemplares en Perú e islas Falkland. En tanto, algunos estatolitos fueron descartados del análisis debido a que no fue posible tomar todas las medidas. Se compararon ejemplares de estaciones diferentes pero con un rango de tamaño y estados de madurez semejantes. Asimismo, una sola persona fue la que realizó todas las medidas. De acuerdo a Pierce et al. (1994), comparar ejemplares muestreados en diferentes épocas introduce menor error que comparar ejemplares medidos por diferentes personas.

Las medidas de los estatolitos de L. gahi de las islas Falkland concuerdan con las descritas por Arkhipkin (1993) y son similares a los examinados por Pineda et al. (1998) en L. gahi de aguas de la costa argentina.

Los resultados del análisis discriminante reflejan diferencias significativas entre los estatolitos de machos y hembras de ambas áreas. El dimorfismo sexual, tanto en las partes blandas como en las estructuras duras, entre machos y hembras ya ha sido descrito anteriormente para cefalópodos por distintos autores (e.g. Cohen, 1976; Hatfield, 1991; Arkhipkin, 1995; Bettencourt et al., 1996; Pineda et al., 1998). Estas diferencias parecen estar asociadas a distintas tasas de crecimiento en ambos sexos, causadas tanto por diferencias individuales en el crecimiento como por diferentes exigencias en la producción de gónada entre machos y hembras (Mangold et al., 1993; Rocha y Guerra, 1999).

Los resultados expuestos señalan que existen diferencias significativas entre las poblaciones del norte del Perú y las de islas Falkland. Esto puede estar indicando que las poblaciones de ambas áreas están aisladas reproductivamente, lo cual es lógico si se tiene en consideración la gran distancia y las diferentes condiciones oceanográficas entre ambas áreas geográficas (Castilla et al., 1993; Fedúlov et al., 1990; Guerrero y Piola, 1997). Los métodos morfométricos no permiten inferir si estas diferencias se deben a influencias del medio ambiente o son, por el contrario, de carácter genético (Ihssen et al., 1981; George-Nascimento y Arancibia, 1992). Por otra parte, Nesis (1987) ha postulado que Loligo gahi que habita las aguas circundantes de las islas Falkland correspondería a otra especie (Loligo patagonica) morfológica y geográficamente diferente a L. gahi, que habita las costas de Perú y Chile. Esta tesis se opone a la de otros autores (Castellanos y Cazzaniga, 1979; Brakoniecki, 1984) que concuerdan en que son la misma especie. Aunque en este trabajo se han hallado diferencias significativas en la morfometría de los estatolitos de ejemplares de ambas zonas, para dilucidar este tema serían necesarios nuevos y más completos estudios, tanto morfométricos como genéticos.

\section{AGRADECIMIENTOS}

Queremos expresar nuestro agradecimiento al Dr. Angel Guerra por sus comentarios y sugerencias al manuscrito de este trabajo. Asimismo al Departamento de Investigación y Desarrollo (DID) de la 
Universidad de Chile por financiar este estudio y a Pesca Chile S.A. por proporcionar las muestras de las islas Falkland.

\section{REFERENCIAS}

Anónimo, 1989. Falkland Islands Interim Conservation and Management Zone. Fish. Rep. 1987/1988, 30 pp.

Arkhipkin, A. 1990. Edad y crecimiento del calamar (Illex argentinus). Frente Marítimo, 6(A): 25-35.

Arkhipkin, A. 1993. Statolith microstructure and maximum age of Loligo gahi (Myopsida: Loliginidae) on the Patagonian shelf. J. Mar. Biol. Ass. U.K., 73: 979-982.

Arkhipkin, A. 1995. Age, growth and maturation of the European squid Loligo vulgaris (Myopsida, Loliginidae) on the West Saharan shelf. J. Mar. Biol. Ass. U.K., 75: 593-604.

Bettencourt, V., L. Coelho, J. Andrade y A. Guerra. 1996. Age and growth of the squid Loligo vulgaris off the south coast of Portugal, using statolith analysis. J. Moll. Stud., 62: 359-366.

Borges, T. 1995. Discriminant analysis of geographic variation in hard structures of Todarodes sagittatus from the North Atlantic. ICES Marine Science Symposium, 199: 433-440.

Brakoniecki, T. 1984. A full description of Loligo sanpaulensis Brakoniecki, 1984 and a redescription of Loligo gahi d'Orbigny 1835, two species of squid (Cephalopoda; Myopsida) from the south west Atlantic. Bull. Mar. Sci., 34: 435-448.

Canavos, G.C. 1996. Probabilidad y estadística aplicaciones y métodos. Editorial McGraw-Hill Interamericana de México S.A., 651 pp.

Castellanos, Z.J.A. de y N. Cazzaniga. 1979. Aclaración a cerca de los Loliginidae del Atlántico Sudoccidental (Mollusca Cephalopoda). Neotropica, 25(73): 59-68.

Castilla, J.C., S.A. Navarrete y J. Lubchenco. 1993. Southeastern Pacific coastal environments: Main features, large-scale perturbations, and Global Climate Change. En: H.A. Mooney, B.I. Kronberg y E.R. Fuentes (eds.). Earth System responses to Global Change. Contrasts between North and South America. Academic Press Inc., pp. 167-188.
Clarke, M.R. 1978. The cephalopod statolith - an introduction to its form. J. Mar. Biol. Ass. U.K., 58: 701-712.

Clarke, M.R., J.E. Fitch, T. Kristensen, T. Kubodera y L. Maddock. 1980. Statoliths of one fossil and four living squids (Gonatidae: Cephalopoda). J. Mar. Biol. Ass. U.K., 60: 329-347.

Clarke, M.R. y L. Maddock. 1988. Statoliths from living species of cephalopods and evolution. En: M. Clarke y E. Trueman (eds.). The Mollusca, Vol. 12. Paleontology and Neontology of Cephalopods. Academic Press, Inc. London, pp. 169-184.

Cohen, A.C. 1976. The systematics and distribution of Loligo (Cephalopoda, Myopsida) in the western north Atlantic, with descriptions of two new species. Malacologia, 15: 299-367.

Cuadra, C.M. 1989. Método de análisis multivariado. Editorial Universitaria de Barcelona, 642 pp.

FAO. 1997. FAO Yearbook of Fisheries Statistics for 1995. FAO, Rome, vol. 80: 1-713.

Fedúlov, P.P., A.V. Remesló, S.N. Burykin y J.A. Pulishchuck. 1990. Variabilidad de la corriente de Malvinas. Frente Marítimo, 6(A): 121-127.

George-Nascimento M. y H. Arancibia. 1992. Stocks ecológicos del jurel (Trachurus symmetricus murphyi Nichols) en tres zonas de pesca frente a Chile, detectados mediante comparación de su fauna parasitaria y morfometría. Rev. Chil. Hist. Nat., 65: 453-470.

Guerra, A. y P. Sánchez. 1985. Crecimiento relativo del estatolito de Sepia officinalis (Cephalopoda, Sepioidea) de la ría de Vigo. Inv. Pesq., 49(4): 545557.

Guerrero, R.A. y A.R. Piola. 1997. Masas de agua en la plataforma continental. En: E.E. Boschi (ed.). El mar argentino y sus recursos pesqueros. Tomo 1: Antecedentes históricos de las exploraciones en el mar y las características ambientales. Instituto Nacional de Investigación y Desarrollo Pesquero, Buenos Aires. pp 107-132.

Gujarati, D.N. 1997. Econometría. McGraw-Hill Interamericana S.A., 824 pp.

Hatfield, E.M.C. 1991. Post-recruit growth of the Patagonian squid Loligo gahi (D’Orbigny). Bull. Mar. Sci., 49(1-2): 349-361. 
Hatfield, E.M.C. y S. Des Clers. 1998. Fisheries management and research for Loligo gahi in the Falkland Islands. CALCOFI Rep., 39: 81-91.

Ihssen, P.E., H.E. Booke, J.M. Casselman, J.M. McGlade, N.R. Payne y F.M. Utter. 1981. Stock identification: Materials and methods. Can. J. Fish. Aquat. Sci., 38: 1838-1855.

Leta, H.R. 1982. Descripción de la morfología de los estatolitos de tres especies de calamar (Cephalopoda: Teuthoidea). Com. Soc. Malac. Uruguay, 6(43): 87-94.

Lipinski, M.A.C., M. Roeleveld y L.G. Underhill. 1993. Comparison of the statoliths of Todaropsis eblanae and Todaropsis angolensis (Cephalopoda: Ommastrephidae) in South African waters. En: T. Okutani, R.K. O'Dor y T. Kubodera (eds.). Recent Advances in Cephalopod Fisheries Biology. Tokai University Press, Tokyo. pp. 263-273.

Mangold, K.M., R.E. Young y M. Nixon. 1993. Growth versus maturation in cephalopods. En: T. Okutani, R.K. O'Dor y T. Kubodera (eds.). Recent Advances in Cephalopod Fisheries Biology. Tokai University Press, Tokyo. pp. 697-703.

Manly, B.F. 1994. Multivariate statistical methods, a primer. Chapman \& Hall, London, 215 pp.

Nesis, K.N. 1987. Cephalopods of the world. Neptune City T.F.H. Publications Inc., 351 pp.
Patterson, K.R. 1988. Life history of Patagonian squid Loligo gahi and growth parameter estimates using least-squares fits to linear and von Bertalanffy models. Mar. Ecol. Prog. Ser., 47: 65-74.

Pierce, G.J., L. Hastie, A. Guerra, R.S. Thorpe, F. Howard y P.R. Boyle. 1994. Morphometric variation in Loligo forbesi and Loligo vulgaris: regional, seasonal, sex, maturity and worker differences. Fish. Res., 21: 127-148.

Pineda, S.E., A. Aubone y N.E. Brunetti. 1996. Identificación y morfometría comparada de las mandíbulas de Loligo gahi y Loligo sanpaulensis (Cephalopoda Loliginidae) del Atlántico Sudoccidental. Rev. Invest. Des. Pesq., 10: 85-99.

Pineda, S.E., D.R. Hernández y N.E. Brunetti. 1998. Statolith comparison of two south-west Atlantic loliginid squid: Loligo sanpaulensis and Loligo gahi. S. Afr. J. Mar. Sci., 20: 347-354.

Rocha, F. y A. Guerra. 1999. Age and growth of two sympatric squid Loligo vulgaris and Loligo forbesi, in Galician waters (NW Spain). J. Mar. Biol. Ass. U.K., 79: 697-707.

Roper, C.F.E., M.J. Sweeney y C.E. Nauen. 1984. FAO Species Catalogue. Cephalopods of the world. An annotated and illustrated catalogue of species of interest to fisheries. FAO Fish. Synop., 125(3): 1-277. 
